\title{
FPGA IMPLEMENTATION OF IMAGE SEGMENTATION BY USING EDGE DETECTION BASED ON SOBEL EDGE OPERATOR
}

\author{
Subodh Kumar ${ }^{1}$, Prabat Pandey ${ }^{2}$ \\ ${ }^{1,2}$ M.Tech, Student, Department of electronics \& Communication Engg., Acropolis Institute of Tech And Research, Indore \\ (M.P),India, Subodh88acropolics@Gmail.Com
}

\begin{abstract}
In this paper, we present the method of "FPGA implementation of image segmentation by using edge detection based on the sobel edge operator". due to advancement in computer vision it can be implemented in fpga based architecture. image segmentation separates an image into component regions and object. Segmentation needs to segment the object from the background to read image properly and identify the image carefully. Edge detection is fundamental tool for image segmentation. Sobel edge operator, which is very popular edge detection algorithms, is considered in this work. Sobel method uses the derivative approximation to find edge and perform 2-D spatial gradient measurement for images uses horizontal and vertical gradient matrices. The fpga device providing good performance of integrated circuit platform for research and development. The compact structure of image segmentation into edge detection can be implemented in MAT LAB using VHDL code and the waveform is shown in the model sim.
\end{abstract}

Keywords: VLSI, FPGA, image segmentation, sobel edge operators, edge detection pixel, mat lab.

\section{INTRODUCTION}

Digital image processing is the use of computing algorithms to perform image processing to digital images .Digital image processing becomes more and more important in The areas of communication, management, remote-sensing, medicine industrial-automation, seismology, robotics , aerospace ,and education.

In digital image processing image segmentation is an essential step for image analysis. The image segmentation separates an image into its components parts and its objects. Segmentation algorithms for image are generally based on the discontinuity and similarity image intensity values. Discontinuity which is based on the abrupt changes in intensity and similarity is based on the partitioning an images into the regions. Edge detection is a part of the image segmentation the effectiveness of many images processing depends upon the perfection of the detecting meaning full edges. It is one of the techniques for detecting intensity discontinuity in digital images. The process of classifying and placing sharp discontinuities in image is called edge detection. These discontinuities are immediate changes into pixel concentration which distinguish boundaries of objects. Here one of the important classical method for edge detection engage convolves the image through operator, which is constructed to large gradient in the image returning values of zero in uniform region. In this paper we present the fpga implementation of image segmentation based on the sobel operator. Field Programmable Gate Array (FPGA) technology has become an alternative for the implementation of software algorithms. the algorithm is simulated in MATLAB, and then the same is implemented into VHDL with the help of Xilinx ISE and the Model sim simulation results are verified with MATLAB results.

\section{MODULES}

\subsection{Image Segmentation}

Image segmentation is very important application in the digital image processing. Image segmentation is the process of partitioning a digital images into multiple regions or sets of pixel. In image partitions are different objects which have the same texture or color. The image segmentation are a set of regions that are entire image together and set of contours extracted from the images. All of the pixel in region are similar with respects to some characteristics such as colors, intensity, or texture. Adjacent region are considerably different with respects to the same individuality. The different approaches of image segmentation are ,finding boundaries between regions based on the discontinuities in intensity levels, Thresholds based on the distribution of pixel properties , such as intensity values and . last is Based on finding the region directly.

Image segmentation is the process of extracting features or regions of interest from an acquired image for further computer analysis. The image is sliced into multiple regions based on some property of the pixels. These properties are intensity, texture, position or some local or global statistical 
parameters. Segmentation using computer vision finds multiple applications especially in the area of biomedicine, communication, management, remote-sensing, medicine industrial-automation, seismology, robotics, aerospace ,and education Typical computer vision applications usually require an image segmentation preprocessing algorithm as a first procedure. At the output of this stage each object of the image represented by a set of pixels is isolated from the rest of the scene. The purpose of this step is that the objects and background are separated into non-overlapping sets.

\subsection{Edge Detection}

Edge detection is important step in digital image processing for image segmentation. it is process of locating an edge of an image detection of edge in an image is very important step to words understanding the image features. Edge consists of meaning full region features and contained significant information it reduces significantly the amount of the image size and preserving the important structural properties images. Since edge occur at image location representing object boundaries, edge detection is extensively used in image segmentation when images are divided into areas corresponding to different objects. In image processing, the edge detection treats the localization of important variaton of a grey level images and the detetction of the physical and geometrical properties of objects of the scence .It is a fundamental process detects and outlines of an objects and boundaries among objects and the background in the image.Edge detection is the most familar apporach for finding or detetcing significant discontinuities in intensity values.

Edge detection is more common for finding detecting discontinuities in grey level than detecting isolated points and thin lines because isolated points and thin lines not occur frequently in most practical images. The edge is the boundary between two region with relatively distinct grey level properties . It is assumed that the transition between two region can be determined on the basis of grey level continuities alone.

\section{IMPLEMENTATION OF EDGE DETECTION}

Edge are characterizes boundaries and the problem of fundamental importance in image processing. Edges in images are areas with strong intensity contrast- a jump in intensity from one pixel to another pixel .The edge of images are considered to be most important attributes that provided valuable information for human perception. The edge detection is a terminology in image processing in the area of features extraction is refer to algorithms which main aim is to identifying point in digital image at which the image brightness changes sharply. The data of edge detection is very large so the speed of image processing is a difficult problem. Sobel operator is commonly used in edge detection.

\subsection{Sobel Edge Detection Operators}

The sobel edge operator a 2-D spatial gradient measurement on an image and emphasizes region of high spatial gradient that corresponds to edges. It is used to find the approximate absolute gradient magnitude at each point in an input grey scale. it is differential of two rows or two column, so the element of the edge on both sides has been enhanced, so that the edge seems thick and bright. The non maximal suppression stage identifies pixel that are local maxima in the direction of the gradient using magnitude and orientation of the pixel. The major orientation of the gradient, either horizontal or vertical is obtained by comparing the individual component, $\mathrm{dx}$ and $\mathrm{dy}$ which are convolving the smoothed image with the derivative of Gaussian which is shown in fig 1

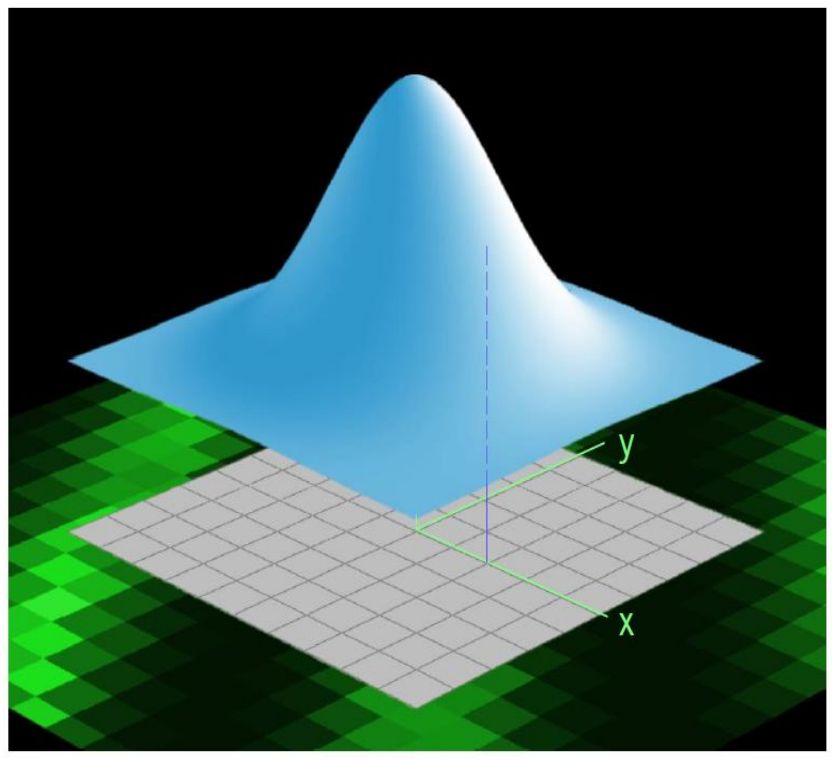

Fig 1.gradient component

In the theory atleast, the operators consists of a pair $3 \times 3$ convolution kernels as shown in fig 2 . one kernel is simply the other rotated by 90 degree. This is very similar to the Roberts cross operators .The convolution mask of the sobel operator are given is shown in fig 2 .

\begin{tabular}{|l|l|l|}
\hline-1 & 0 & 1 \\
\hline-2 & 0 & 2 \\
\hline-1 & 0 & 1 \\
\hline
\end{tabular}

$\mathrm{X}$ direction

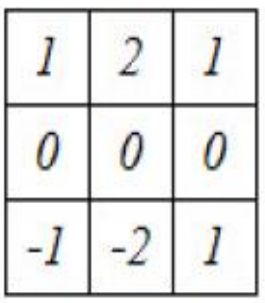

Y direction
Fig 2 Convolution kernel in $\mathrm{X}$ and $\mathrm{Y}$ direction 
These kernel can be combined together to find the absolute magnitude of the gradient at each point. The gradient magnitude is given by :

$$
\begin{aligned}
& |\nabla f|=\sqrt{G_{x}^{2}+G_{y}^{2}} \\
& |\nabla f|=\left|G_{x}\right|+\left|G_{y}\right|
\end{aligned}
$$

\subsection{Gradient Operator}

Gradient-based edge detection is the most common approach for detecting meaningful discontinuities in gray level. The most common type of edge detection process uses a gradient operator. Gradient is a vector having both magnitude as well as direction. The gradient of an image $f(x, y)$ at location $(x, y)$ is defined as vector. The gradient vector points in the direction of maximum rate of change of $f$ at coordinates $(x, y)$. An important quantity in the edge detection is magnitude of this vector.

The gradient of na image $\mathrm{F}(\mathrm{x}, \mathrm{y})$ at location $(\mathrm{x}, \mathrm{y})$ is the vector

$$
\nabla f=\left[\frac{G_{x}}{G_{y}}\right]=\left[\frac{\partial f / \partial x}{\partial f / \partial y}\right]
$$

The gradient vector points in the dircetion of maximum rate of change of $\mathrm{f}$ at $(\mathrm{x}, \mathrm{y})$.In edge detection, An important quantity is the maggnitude of the this vector:

$$
|\nabla f|=\sqrt{G_{x}^{2}+G_{y}^{2}}
$$

The gradients take its maximum rte increase of $\mathrm{f}(\mathrm{x}, \mathrm{y})$ per unit distance in the distance in the direction of $\nabla f$.

The gradient magnitude is commonly approximated by:

$$
|\nabla f|=\left|G_{x}\right|+\left|G_{y}\right|
$$

This is simpler to implement .The diretcion of the gradient vector is also important and is given by

$$
\alpha(x, y)=\tan ^{-1}\left(\frac{G_{y}}{G_{x}}\right)
$$

\subsection{FPGA HARDWARE IMPLEMENTATION}

The field programmable gate array implementation of edge detection is shown in fig respectively. This design uses $3 \times 3$ convolution kernels processing 500x500 Gray Scale Image from the database in the personal computer. The architecture is shown in Fig 3. This system is divided into basic four modules: 3 x 3 pixel generation modules, Sobel enhancement operator module, edges control module and binary segmentation. In this system, there are various signal are :Clk is the clock signal, Data input is the pixel signal of Gray Scale Image, Result is the result of edge detection operator signal, Generation data and Data are the middle signal.

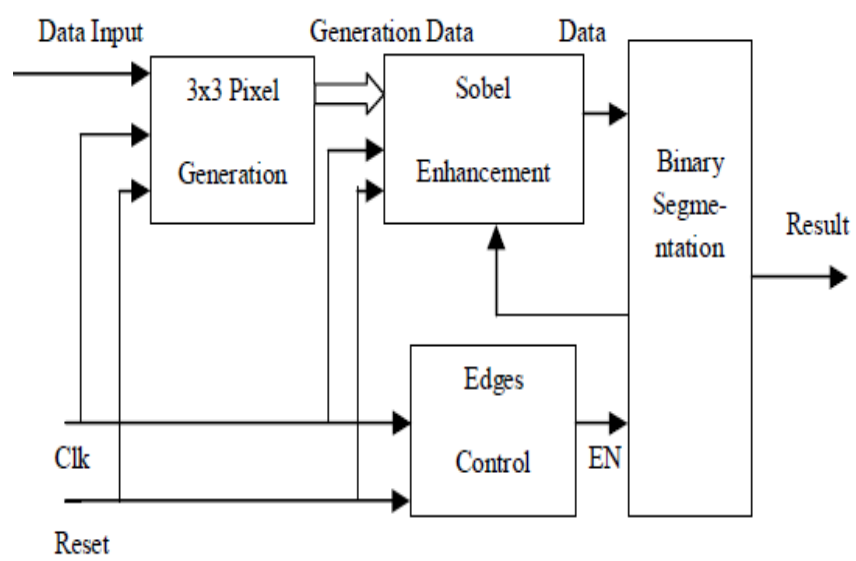

Fig 3 Architecture

\section{IMPLEMENTATION OF IMAGE SEGMENTATION USING THE SOBEL OPERATOR:}

The Image segmentation is a very important application in the field of image processing. Image segmentation is the process of extracting features or regions of interest from an acquired image for further intelligent computer analysis. The image is sliced into multiple regions based on some property of the pixels. These properties are intensity, texture, position or some local or global statistical parameters. There are number of literatures on image segmentation both semiautomatic and automatic.

\subsection{Segmentation Algorithms Based On the Edge Detection:}

Here the segmentation algorithms with the help of the edge detection sobel operator are described in the following steps:

Step 1: Read input image

Step 2: Apply into horizontal mask Gx and vertical mask Gy to the input image.

Step 3: Apply into different sobel edge detection algorithms and find the gradient 


$$
|\nabla f|=\left|G_{x}\right|+\left|G_{y}\right|
$$

Step 4: Construct separate image for Gx and Gy

Step 5: Results are combined to find the absolute magnitude of the gradient.

$$
|\nabla f|=\sqrt{G_{x}^{2}+G_{y}^{2}}
$$

Step 6: The absolute magnitude is the output slope magnitude image.

Step 7: For some slope magnitude images, the pixels values are too small or too high. To improve visibility of those images, scaling has to be done .For small values, it has to be scaled up by appropriate factor. For large values, it has to be scaled down by appropriate factor.

\subsection{Segmentation Architecture Using Sobel Operator}

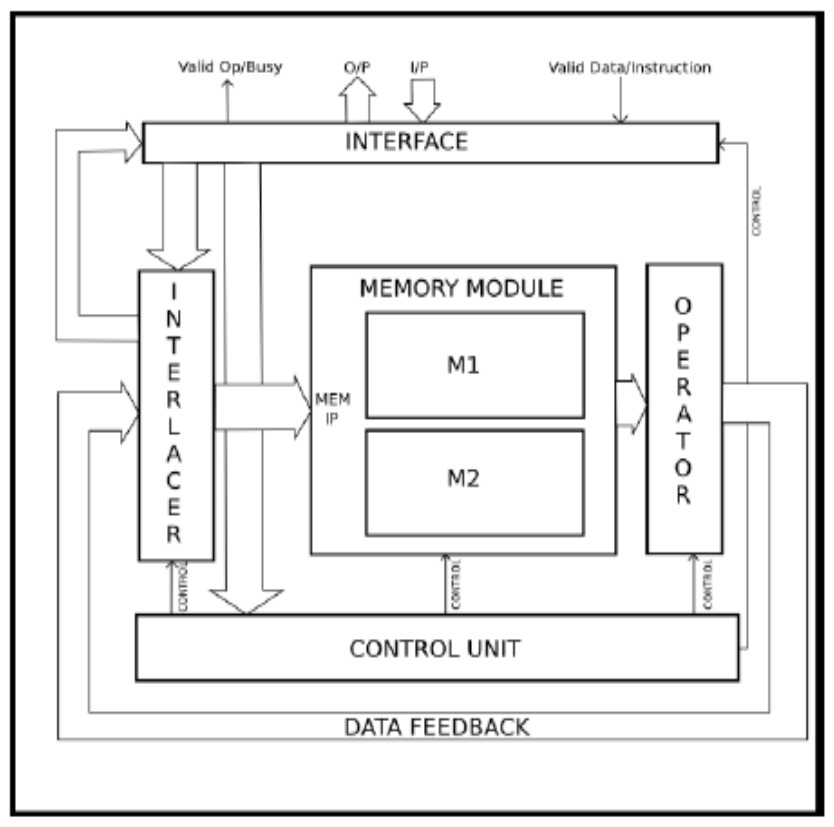

Fig 4 segmentation processor architecture

The proposed segmentation architecture has the shown in the fig 4.It consists of the interface, memory, operator, control unit. In this diagram, the different functional unit described the control unit monitors all the activities of the processor. in this system the control signal are issued to the memory unit and the interface. The memory is stores the image pixel for the processing and the processed image is stored in the read memory section. The input is connected to the write memory and the output is connected with read memory. operator unit perform the edge detection methods for the image segmentation processing. Operator performs the algorithms for the edge detection for the segmentation.

\section{EXPERIMENTAL RESULTS:}

The experimental results for the image segmentation for edge detection in MAT LAB are shown in fig 5. (a)is the input images and the fig 5(b)shows the output images and the simulation waveform for the modules is also shown in the fig 6.
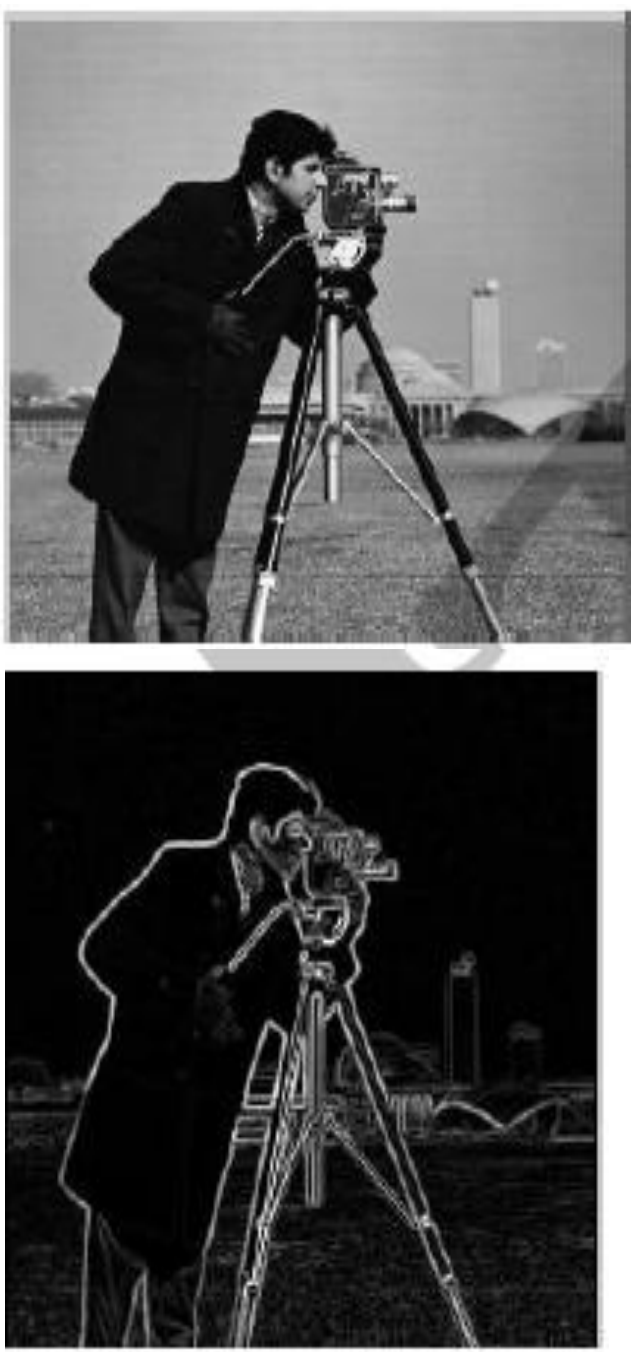

Fig5. Edge detection in mat lab

Here another fig which is shown below of the simulation waveform for iamge segmanttaion using sobel edge operator fig 6(a) image segmentation 6(b) image segmentation using edge operators waveform. 


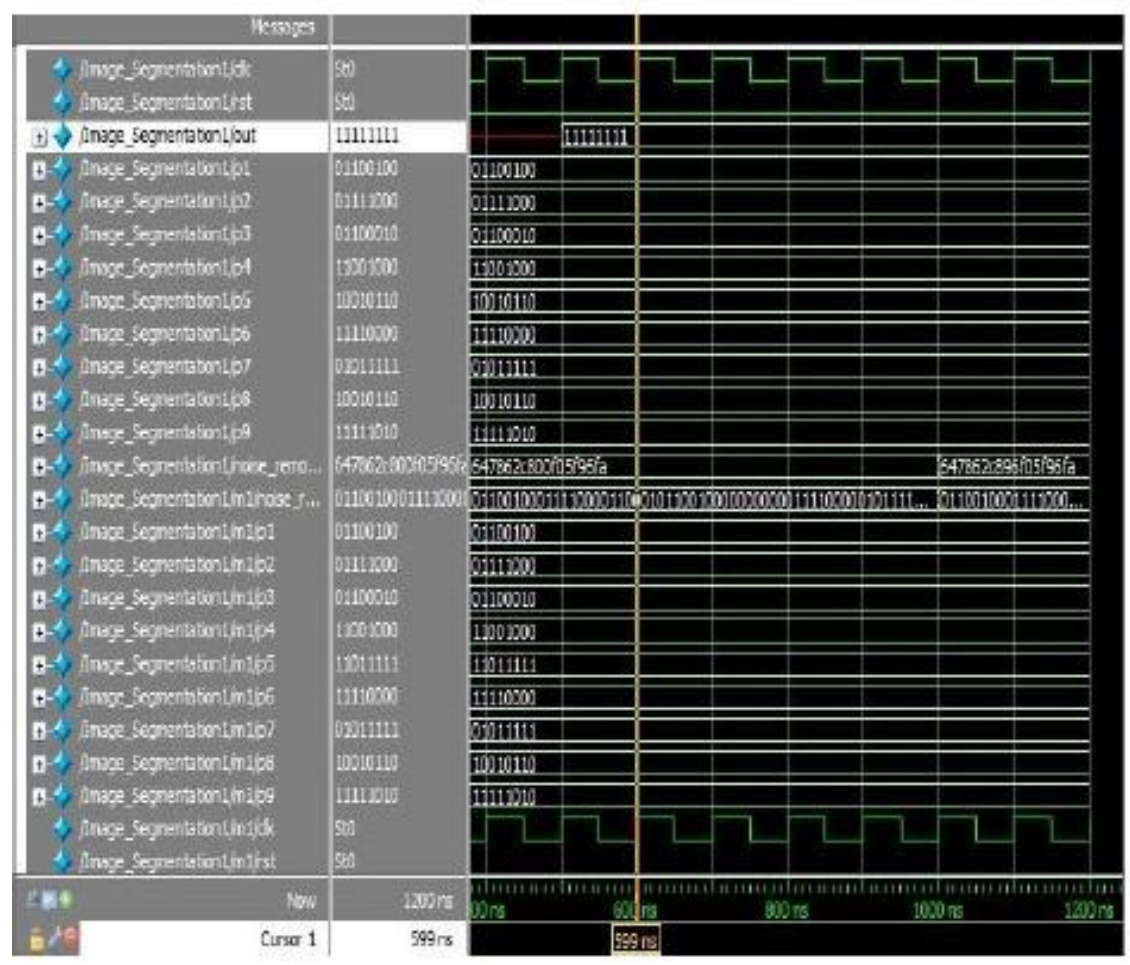

Fig 6(a) segmentation

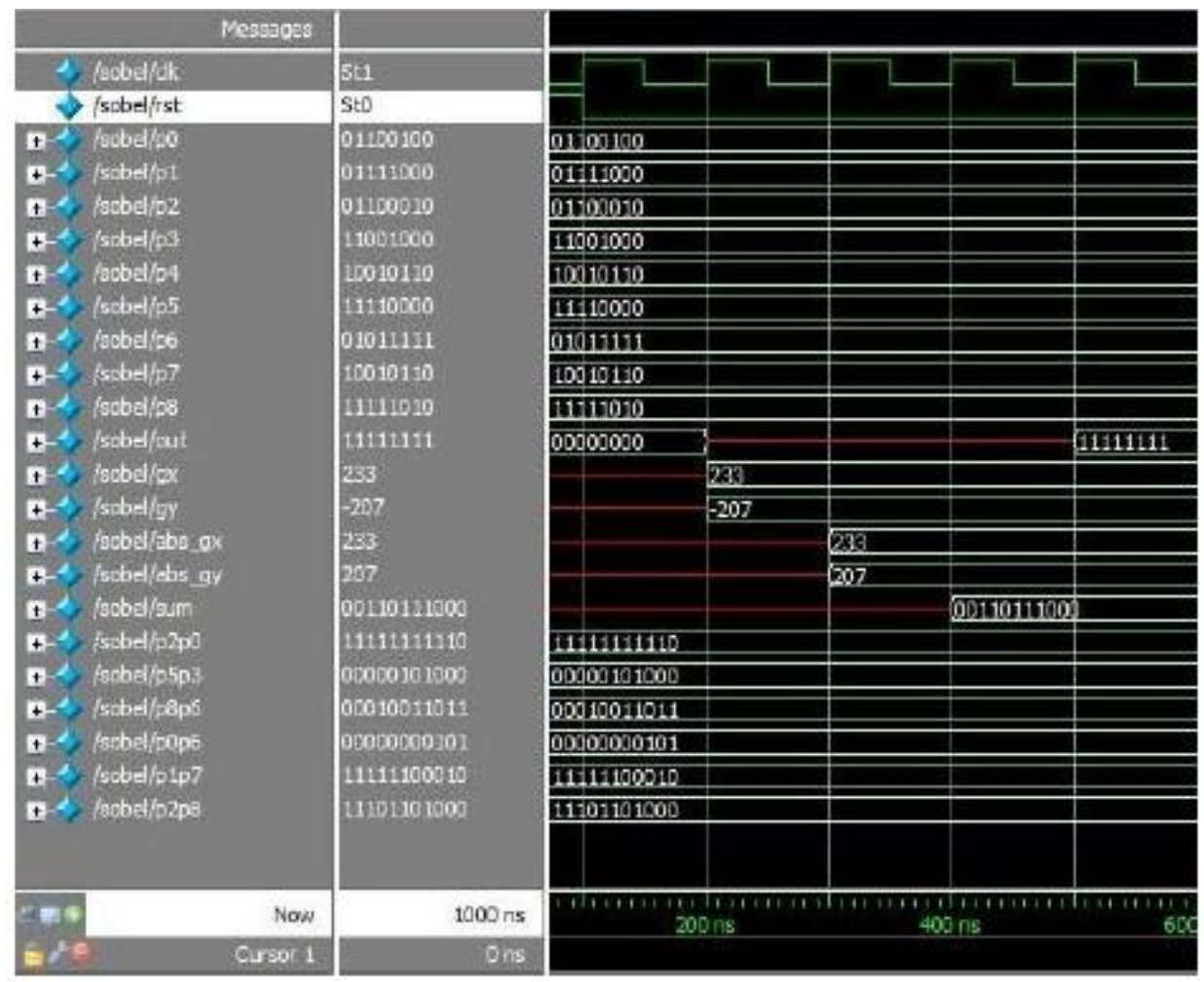

Fig6 (b) segmentation using sobel edge operator 


\section{CONCLUSIONS}

This paper describes the image segmentation techniques using edge detection based on the sobel edge operator and discusses in detail the edge detection Techniques of sobel edge operator and their evaluation. It gives an algorithm which is a combination of detection and evaluation of the edge detectors of the segmentation. The results show that the edge detection in the mat lab and the simulation waveform implemented in the model sim. The fpga based architecture is good and stable techniques for the edge detection.

\section{REFERENCES}

[1] Zdenek Vasicek, Luk as Sek anina, "Novel Hardware Imp lementation of Adaptive Median Filters", 978-1-4244- 2277-7. [2] Hong Shan Neoh, Asher Hazanchuk, "Adap tive Edge Detection for Real-Time Video Processing using FPGAs, Altera Corporation, Innovation Drive.

[3] SOBEL, I., An Isotropic $3 \times 3$ Gradient Operator, Machine Vision for Three - Dimensional Scenes, Freeman, H., Academic Pres, NY, 376-379, 1990

[4] Prewitt, Sobel and Scharr gradient 5x5 convolution matrices Guennadi (Henry) Levkine Email: hlevkin at yahoo.com Vancouver, Canada. First draft,February 2011 Second Draft, June 2012.

[5] A Descriptive Algorithm for Sobel Image Edge Detection, O. R. Vincent, O. Folorunso Proceedings of Informing Science \& IT Education Conference (InSITE) 2009.

[6] Sobel Edge Detection Method For Matlab, Elif AYBAR Anadolu University, Porsuk Vocational School

\section{BIOGRAPHIES}

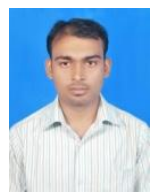

Subodh kumar, B.Tech from Cambridge institute of technology Ranchi, Ranchi university, pursuing $\mathrm{m}$. tech from acropolis institute of technology and research Indore, Madhya Pradesh, India. 